\title{
AN EFFICIENT SUPERVISED MACHINE LEARNING MODEL APPROACH FOR FORECASTING OF RENEWABLE ENERGY TO TACKLE CLIMATE CHANGE
}

\author{
DRUMIL JOSHI, FAWZAN SAYED, JAI BERI \& RANJUSHREE PAL
}

Electronics and Telecommunication Engineering, Dwarkadas J. Sanghvi College of Engineering, Mumbai, India

\begin{abstract}
This paper aims to introduce a reliable forecasting model for the consumption of electricity using renewable sources (namely: offshore wind, onshore wind and solar power) in EU countries, based on live data from the ENTSOE transparency platform as its input. The primary use behind this data science and machine learning methodology, is to help judge the availability of renewable energy resources. Aforementioned software is put to work by inputting desired country and associated parameters. It learns by carefully observing past patterns and their seasonality to make accurate predictions for the future. The ML algorithms used in this process are linear regression, extra trees regression, random forest regression, support vector machine (SVM) and gradient boosting, and precision is substantiated by getting a minimal Symmetric Mean Absolute Error (SMAPE) of 1-2.
\end{abstract}

KEYWORDS: forecasting model, electricity, renewable, ENTSOE, data science, machine learning, SMAPE

Received: Feb 16, 2021; Accepted: Mar 04, 2021; Published: Mar 15, 2021; Paper Id.: IJCSEITRJUN20213

\section{INTRODUCTION}

The irresponsible use of non-renewable natural resources for energy creation in the past century calls for a serious shift in choice of source of electricity for mankind. Coal and natural gas, which are our primary dependencies today, are expected to completely vanish by 2060 if alternative schemes are not considered. However, the projections of the process of moving over to green energy suggest that this problem is not being taken seriously enough, especially when only $10 \%$ of electric utility companies are prioritizing renewable energy.

Another dimension of the problem with non-renewable sources is the dangerous impact of greenhouse gases released into the environment, which lead to global warming and climate change causing health problems, heatwaves, food security complications and many more severe issues worldwide. With a global rise of temperature of $1.1^{\circ} \mathrm{C}$ above pre-industrial level, the last decade was the warmest ever on record: and one of the largest contributors to this problem, are fossil fuel power stations.

The demand for global power is rising exponentially every day. Hence, the countries endeavoring to actively reduce their dependence on natural resources face a trade-off between economic development and the depletion of fossil fuels. But the intensity of this trade-off can be mitigated by realising the immense potential for the green electricity market, due to its significant drop in monetary investment over the past few years, making it a considerable alternative.

Various green energy sources like biomass, geothermal and hydroelectric power can be considered while thinking about the future. However, most countries are focusing on solar and wind energy, as they are the alternatives which have least constraints and limitations. Due to their flexible nature, they are also called as variable 
renewable energy sources or VREs for short. VRE integration with the electric grid is difficult due to its fluctuating nature of output. Forecasting the implementation of the same over a timeframe allows for efficient and systematic planning and electricity scheduling.

The IPCC (Intergovernmental Panel on Climate Change) suggests that the world must become carbon-neutral by 2050 to have any hope of limiting global warming to $1.5^{\circ} \mathrm{C}$. Thus, this paper offers a solution to track the progress in the use of renewable sources in European Union countries over desired parameters to aid in decision-making for industries. It is performed by forecasting consumption using Streamlit, a Python framework efficient for data visualizations and machine learning models and the python library sktime.

\section{Literary Survey}

The data that we use is taken from ENTSO-E's Restful API. ENTSO-E stands for European Network of Transmission System Operators for Electricity. There are 42 electricity transmission system operators (TSOs) spread across 35 countries and ENTSO-E helps coordinate the cross-border system operations, system development and electricity market activities. The Transparency program is an initiative of the ENTSO-E to make all the collected data available to everyone so as to help in the overall growth of the market and to also let the people be aware and scrutinize the activity of the industry. The Transparency Platform restful API is one of the ways through which the real-time data can be accessed for specific locations over a certain period of time. The renewable energy plant data is what we extract using the API and apply machine learning algorithms to this.

\section{METHODOLOGY}

\section{A. Creating of Environment}

Out of the 35 countries, we have only considered 27 of the major countries and so, a JSON file containing the list of the country names and their country code is prepared. This will make it easy to access the country codes and also to add or update the list if required in the future.

The program is written on the python 3.7 platform. It is the leading platform for developing programmers which also contains advanced features for machine learning and data analysis. Sklearn is the machine learning library included in python which contains various tools, models and evaluation techniques. Sktime is an open-source Python toolbox for machine learning with time series. Sktime extends and the scikit-learn API to time series tasks. It provides the necessary algorithms and transformation tools to efficiently solve for time series regression, forecasting, and classification tasks. The library includes dedicated time series learning algorithms and transformation methods not readily available in other common libraries.

\section{B. Analysis of Live Data}

The first program that is run takes the country code as input (taken from the JSON file) in its function argument. The data from this country is taken from the current time up to the data from one month ago. This data is then resampled to have a sampling frequency of 1 hour to make the outputs consistent across all the machine learning models.

The second program again is divided into three functions. The first function calls the required machine learning algorithm from the sklearn library according to what the user wants to apply and makes a variable of the respective type. The second function takes the dataframe created by the first program and divides the data into training data and testing 
data. The machine learning model is then trained using the training data. The third function calculates the SMAPE value of the trained model using the predicted and testing values.

\section{Machine Learning Algorithms Used}

There are six models from which the user can select:

- Linear Regression- Regression is a basic mathematical operation including several modelling and analysis techniques. Regression analysis is a tool which explains the relation between the independent variables against its dependencies. The dependent variable is indicated by the $\mathrm{Y}$, and the independent variables by $\mathrm{X}$ [1].

- Random Forest- Random Forests is one of supervised learning algorithm. The forest contains trees. Random Forest is a combination of $\mathrm{N}$ number of decision trees. Random forests form decision-making trees in randomly selected data samples, get predictions for each tree and select the best solution by voting [2]. Unplanned forests are considered to be the most accurate and powerful method due to the number of decision trees that participate in the model development. It prevents the model from overcrowding.

- Gradient Boosting- Boosting builds models by turning weak learners into strong learners in an iterative way. Increasingly, individual models are not built on random subsets of data and features but respectively by placing more weight on cases with incorrect predictions and high errors [3]. While the AdaBoost model identifies the shortcomings by using high weight data points, gradient boosting performs the same by using gradients in the loss function.

- Support Vector Machine- Support Vector Machine (SVM) is a simple Directed Machine Learning Algorithm used for separation. SVM detects a hyper-plane that creates a boundary between data types. In 2-D space, this hyperplane is nothing but a line. In SVM, we organize each data object in a database in the N-dimensional space, where $\mathrm{N}$ is the number of objects / attributes in the data. Next, we find a hyperplane suitable for separating details. So, by this, you should have understood that naturally, SVM can only make binary divisions (i.e., choose between two classes).

- Extra Trees- Extremely Unplanned Tree Separation (Extra Trees) is an integrated learning approach that combines the effects of multiple unrelated decision trees collected in the "forest" to produce their own classification effect. It is very similar to the Random Forest Classifier and differs only in the way trees are cut down in the forest. Each decision tree in the forest of additional trees is built from the original training sample. Then, at each test site, each tree is given a random sample of features $\mathrm{k}$ from the set where each decision tree should select the best data classification feature according to specific mathematical terms (usually the Gini Index). This random sample of symptoms leads to the formation of many trees of incompatible decisions.

- XGBoost- XGBoost represents stands for Extreme Gradient Boosting Expansion; the exact implementation of the Gradient Stimulation method which uses a more accurate measurement to obtain a good tree model. It uses a number of advanced techniques that make it particularly effective, especially for systematic details. Creating second-order gradients, that is, the release of the second part of the loss function that provides more details about the direction of the gradients and how you can get to the minimum of our loss function [4]. While standard gradient enhancement uses the loss of our basic model (e.g., decision tree) as a proxy to minimize the error of the 
entire model, XGBoost uses second-order acquisition as a measure and improved familiarity, which improves model performance.

Table 1:Algorithm Comparison

\begin{tabular}{|l|c|c|c|c|}
\hline \multicolumn{1}{|c|}{ ML Algorithm } & Power & Interpretability & Raw Implementation & Normalization \\
\hline Linear Regression & Low & Good & Easy & No \\
\hline Extra Trees Regression & High & Good & Difficult & No \\
\hline Random Forest Regression & High & Good & Difficult & No \\
\hline Support Vector Machine & High & Medium & Very Difficult & Yes \\
\hline Gradient Boosting & High & Medium & Medium & Yes \\
\hline XGBoost & High & Medium & Difficult & Yes \\
\hline
\end{tabular}

\section{Deployment}

Streamlit is an open-source Python library that makes it easy to create and share beautiful, custom web apps for machine learning and data science. In our project we have customized the entire web app by creating options to choose the EU country, the Machine Learning algorithm to be applied on the live data and the time span and window length on which the forecasting has to be done. The renewable energy which will be forecasted will be Wind Onshore Energy, Wind Offshore Energy and Solar Energy. The renewable energy depends on the selected country and its participation in the climate change. The name of the EU countries has been provided by creating a JSON file as described above. The live data is being passed through the Reduced Regression Forecaster for forecasting. The live data is been displayed using the function of area chart of the streamlit framework, then the forecasted values are displayed line charts of the streamlit platform [5]. The accuracy metric used here is SMAPE. Symmetric means total error measurement accuracy based on percentage (or related) errors. It is usually defined as follows:

$$
\operatorname{SMAPE}=\frac{1}{n} \sum_{t=1}^{n} \frac{\left|F_{t}-A_{t}\right|}{\left(A_{t}+F_{t}\right) / 2}
$$

where $A_{t}$ is the actual value and $F_{t}$ is the forecast value.

The total difference between At and Ft Ft is half divided by the sum of the real At $A_{t}$ values and the $F_{t}$. The value of this number is summarized in all relevant points $t$ and then divided by the number of points entered $n$. Depending on the accuracy of the data, a better measure of the relative accuracy can be obtained based on the log of the accuracy measure: $\log \left(\mathrm{F}_{\mathrm{t}} / \mathrm{A}_{\mathrm{t}}\right)$ When used in constructing payable forecasting models it is similar to the geometric definition.

A sample output of Web app is as shown below:

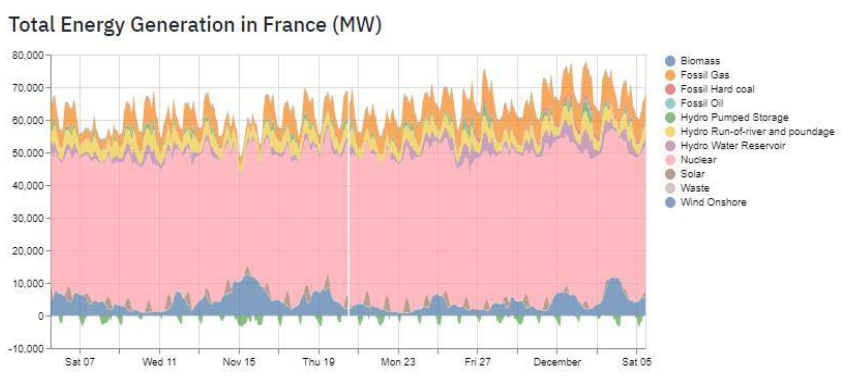

Figure 1: First output from the App 


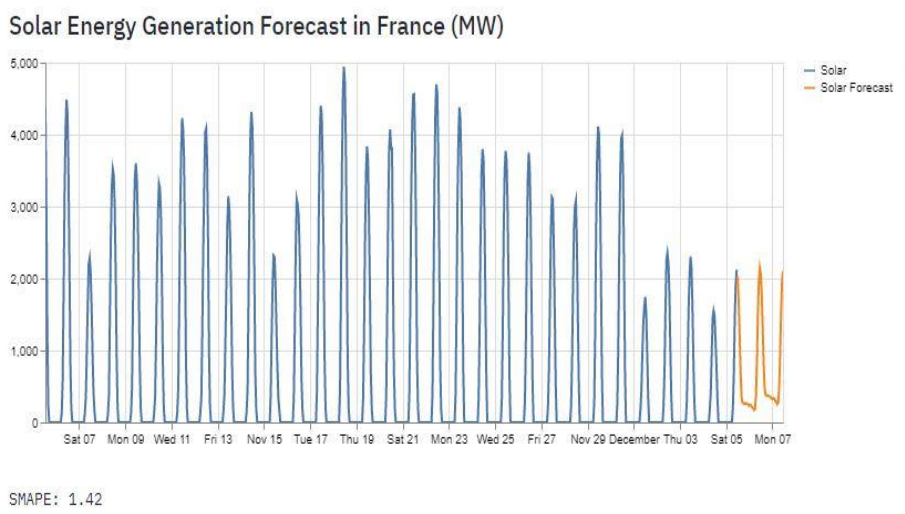

Figure 2: Second output from the App

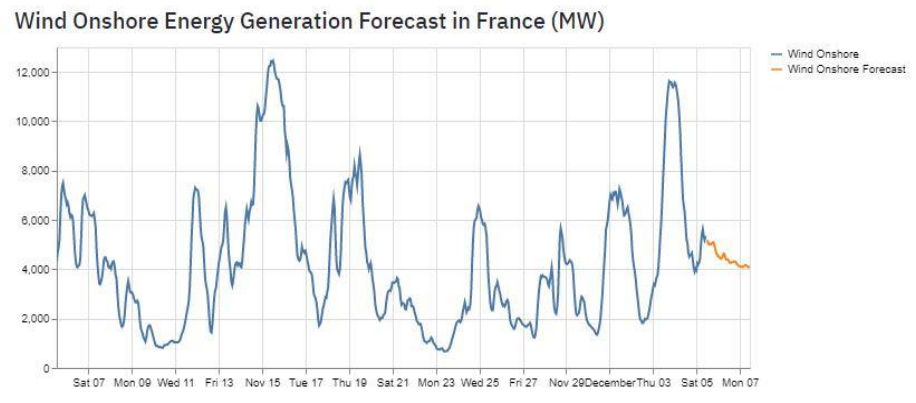

SMAPE: 0.23

Figure 3:Third output from the App

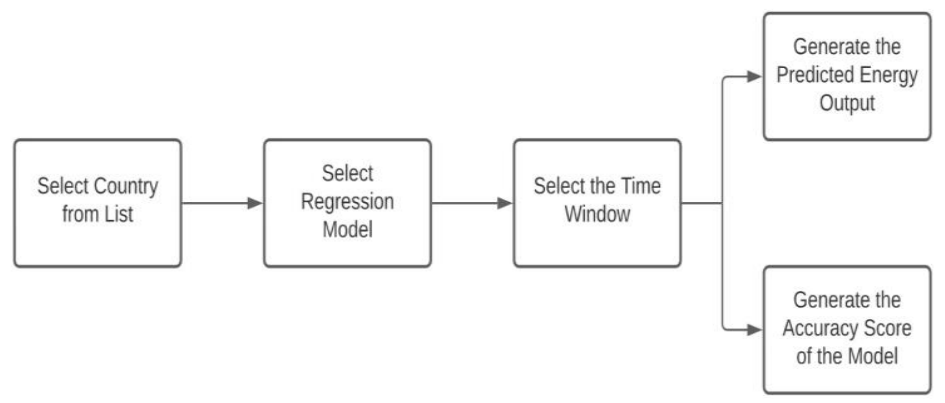

Figure 4: Flowchart of the App

\section{RESULTS}

The entire project has been deployed with the help of streamlit and a web app has been created. The web app has direct access to the live data of the ENTSOE transparency platform via the API key and thus interactive graphs are being plotted of the total and different types of energy consumption. As the main motive of the given research is to find the forecasting of renewable energy resources, thus the user has to select the country and the axioms (in hours) to find the forecasted value. The forecasted values are a result of past seasonal and cyclic patterns and are represented by the orange line. The accuracy metric here considered was SMAPE which stands for Symmetric Mean Absolute Error. The respective models are trained in such a way that the SMAPE value remains at least.

Considering 3 EU Countries we get the metrics for the corresponding renewable energy forecast as follows: 


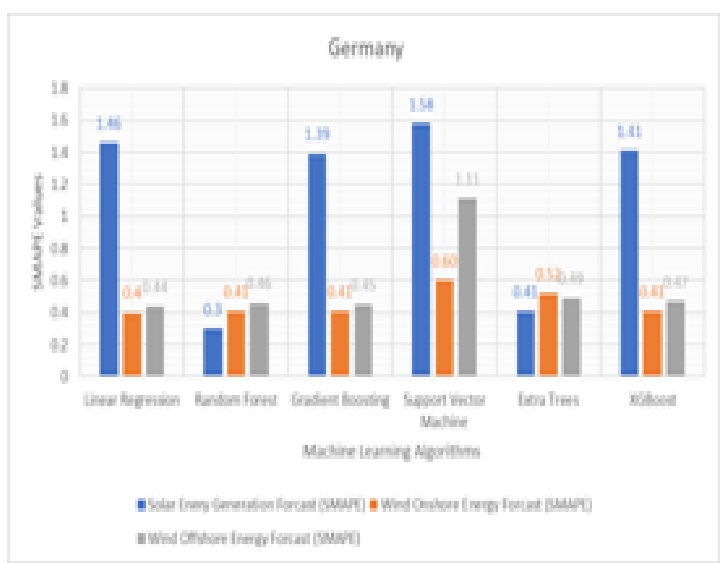

Figure 5: SMAPE Values for Germany

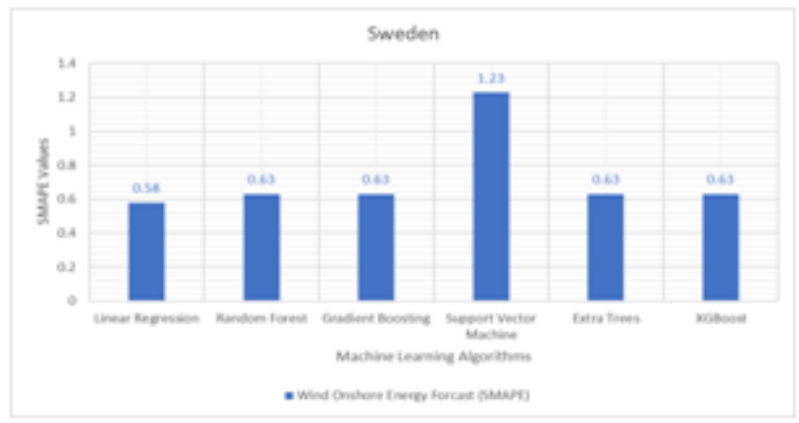

Figure 6: SMAPE Values for Sweden

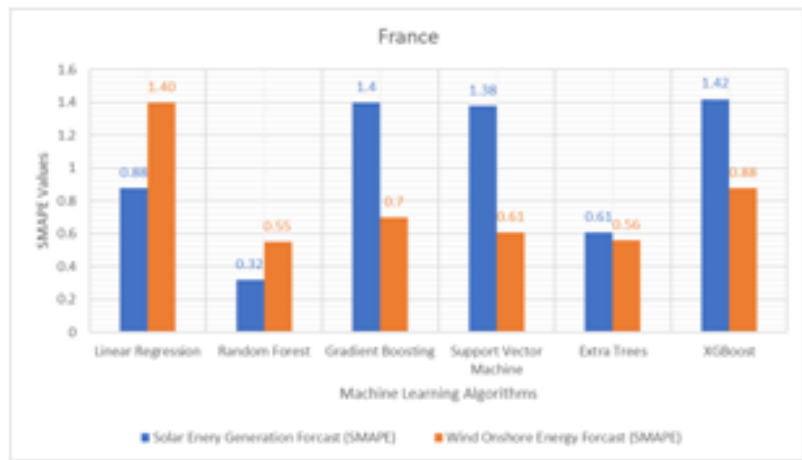

Figure 7: SMAPE Values for France

\section{CONCLUSIONS}

In this research paper, we have used Data science and Machine learning technologies to forecast live data from the ENTSOE transparency platform. We have mentioned the step-by-step procedure to analyze the data and forecasting the renewable energy for the required EU country and the required time span. Hence, we calculated the performance of each model using accuracy metric known as SMAPE. This problem is basically a regression problem and the algorithm which outperforms in this situation is Random Forest and Extra Trees which consistently keeps SMAPE value in the range of 1-2. The system uses API to extract the data and also makes optimal use of machine learning algorithms which satisfies the customer by providing accurate output and preventing the risk of forecasting in the renewable energy sources. Indeed, we'll be encouraged to learn more about Artificial Intelligence and its influence in saving the environment. 


\section{REFERENCES}

1. Baltes, B. B., Parker, C. P., Young, L. M., Huff, J. W., \& Altmann, R. (2004). The practical utility of importance measures in assessing the relative importance of work-related perceptions and organizational characteristics on work-related outcomes. Organizational Research Methods, 7(3), 326- 340. doi: 10.1177/1094428104266014

2. Breiman, L. (2001b). Random forests. Machine Learning, 45, 5-32.

3. Friedman JH (2001). “Greedy function approximation: a gradient boosting machine.” Annals of Statistics, pp. $1189-1232$.

4. Friedman J, Hastie T, Tibshirani R, et al. (2000). "Additive logistic regression: a statistical view of boosting (with discussion and a rejoinder by the authors).” The annals of statistics, 28(2), 337-407.

5. A. Bifet, G. Holmes, B. Pfahringer, R. Kirkby, and R. Gavaldà, "New ensemble methods for evolving data streams," in KDD, 2009, pp. 139-- 148 
